\title{
International Conference and Exhibition on
}

\section{Marine Drugs and Natural Products}

July 25-27, 2016 Melbourne, Australia

\section{A biomedical investigation on the hepatoprotective effect of Coriolus versicolor $L$ and Salvia miltiorrhiza Bge}

\author{
Ming Hong and Ning Wang \\ The University of Hong Kong, China
}

Background: Chinese herbal Medicine exhibits hepatoprotective effect on various experimental models. Coriolus versicolor L and Salvia miltiorrhiza Bge are two widely used Chinese herbal Medicine to treat liver injury in the clinical practice of Chinese Medicine practitioners. However, the experimental evidence on its hepatoprotective effect and its major active component are still not fully understood.

Methods: Experimental dosage is estimated according to the human dose of "Liver" formula composition. We will (a) establish the alcohol-induced liver injury model by pre-treating or post-treating ethanol to mice and investigate the action of Coriolus versicolor $\mathrm{L}$ and Salvia miltiorrhiza Bge on alcohol induced acute liver injury in mice; (b) establish the alcoholic chronic liver injury model by feeding the liquid ethanol diet to mice and determine the protective effect of Coriolus versicolor $\mathrm{L}$ and Salvia miltiorrhiza Bge on alcoholic chronic liver diseases in mice. And (c) establish the non-alcoholic chronic liver injury model by feeding choline-deficiency, amino acid-defined (CDAA) diet and injecting carbon tetrachloride to mice and determine the protective effect of Coriolus versicolor $\mathrm{L}$ and Salvia miltiorrhiza Bge on non-alcoholic chronic liver diseases in mice. And (d) the related mechanism of Coriolus versicolor $\mathrm{L}$ and Salvia miltiorrhiza Bge on alcoholic and non-alcoholic liver disease will be investigated. The role of oxidative stress in mediating the pathological process of alcoholic and non-alcoholic liver diseases will be focused.

Findings \& Prospects: Successful elucidation on the action and mechanism of the hepatoprotective effect of the major active component of Coriolus versicolor $\mathrm{L}$ and Salvia miltiorrhiza Bge will provide the experimental evidence on its clinical use as a healthy supplement for patients with hepatic problem, and facilitate re-discovery of Coriolus versicolor L and Salvia miltiorrhiza Bge to develop active components with greater potency in liver protection.

\section{Biography}

Ming Hong is a PhD student in School of Chinese Medicine, Li Ka Shing Faculty of Medicine, The University of Hong Kong. His research is mainly focused on liver disease and Chinese herbal medicines. He has published 3 papers in reputed journals during his PhD study.

\section{Notes:}

